\title{
Análisis de los antecedentes motivacionales en árbitros de baloncesto y su incidencia en el agotamiento emocional
}

\author{
Analysis of the motivational antecedents in basketball \\ referees and its incidence on emotional burnout
}

\author{
Inmaculada González-Ponce, Pedro A. Sánchez-Miguel, Diana Amado, José M. Chamorro y Juan José Pulido
}

\begin{abstract}
PALABRAS CLAVE: Autodeterminación, deporte, burnout, necesidades psicológicas

RESUMEN: El objetivo de este estudio fue analizar las relaciones entre los antecedentes motivacionales y su influencia en el burnout en árbitros de baloncesto. Para ello, se han seleccionado 30 árbitros con edades comprendidas entre los 17 y 41 ańos $(M=24.8 ; D T=5.74)$. Los resultados revelaron relaciones positivas entre los mediadores de la frustación de las necesidades psicológicas básicas (autonomía, competencia y relaciones sociales) con la desmotivación. Lo mismo sucede con el agotamiento emocional que se asocia de forma positiva y significativamente con la frustación de autonomía, competencia y relaciones sociales. Además, el mayor predictor del agotamiento emocional fue la desmotivación. Finalmente, los resultados son
\end{abstract}

discutidos para mejorar el conocimiento sobre la motivación y el burnout. KEYWORDS: Self-determination, sport, burnout, psychological needs ABSTRACT: The aim of this study was to examine the relationships between motivational antecedents and its influence on burnout in basketball referees. Hence, 30 referees ranging in age from 17 to 41 years old $(M=$ 24.8; $S D=5.74)$ were selected, Results revealed a positive relationship between basics psychological needs thwarting (autonomy, competence and relatedness) and amotivation. Moreover, emotional burnout was positive related with autonomy, competence and relatedness thwarting. Furthermore, emotional burnout emerged as the strongest predictor of amotivation. Finally, results are discussed in the light of enhance the knowledge about motivation and burnout.

\section{Introducción}

En el deporte escolar, existen muchas personas que influyen de forma directa en el desarrollo integral del joven deportista (Montero, Ezquerro, y Buceta, 2005) como son los padres (Torregrosa, Cruz, Sousa, Villadrich, Villamarín, GarcíaMas, y Palou, 2007) y los entrenadores (Feu, Ibáńez, Graça, y Sampaio, 2007). Sin embargo, escasos estudios se han preocupado de analizar la importancia de los árbitros deportivos en categorías de formación y sus regulaciones motivacionales para desempeñar la función de que se cumplan las reglas deportivas

Además, es relevante conocer cuáles son las inquietudes que llevan a un árbitro a dedicar tiempo a la actividad que desempeña, en concreto, qué regulación motivacional le impulsa a llevar a cabo esta función. Por ello, el presente trabajo, al igual que otras investigaciones del contexto deportivo con otros colectivos (Sánchez-Oliva, Leo, Sánchez-Miguel, Gómez, y García-Calvo, 2011), se basa en la Teoría de la Autodeterminación (TAD) para analizar los antecedentes motivacionales de los árbitros de baloncesto (Deci y Ryan, 2000), la cual establece que hay tres tipos de motivación en lo que respeta al nivel de autodeterminación. En el primer nivel, la

Dirección para correspondencia: Inmaculada González Ponce. Departamento de Didáctica de la Expresión Musical, Plástica y Corporal. Universidad de Extremadura. Avenida de la Universidad, S/N, 10071, Cáceres.

Email: ingopo@unex.es motivación intrínseca, en el que una persona se dedica a desarrollar cualquier acción por el simple placer que le produce la actividad, siendo un fin en sí misma (Deci y Ryan, 1985).

En el segundo nivel de autodeterminación se encuentra la motivación extrínseca, en la que el árbitro tiene una regulación ajena a la propia actividad, subdividida en regulación integrada, identificada, introyectada y externa. Los árbitros con una regulación integrada, se caracterizan porque la propia actividad está inmersa en su estilo de vida (Deci y Ryan, 2000). Este tipo de motivación no sólo implica la identificación con la importancia de la actividad, sino también con el deseo de integrar este sentido de identificación, siendo un aspecto más de la propia cotidianeidad del individuo. La regulación identificada ocupa el segundo lugar dentro de la motivación extrínseca, en la que el árbitro percibe que la actividad que lleva a cabo es favorable, encontrando beneficio personal por el hecho de realizarla. Seguidamente, el tercer nivel de motivación extrínseca se denomina introyectada. Es un constructo que se define por el intento de evitar los sentimientos negativos como la culpa y la ansiedad, así como a la experiencia positiva del ego relacionadas con sentimientos como el orgullo. En el último eslabón de la motivación extrínseca, encontramos la regulación externa, que lleva a un sujeto a desarrollar cualquier actividad por el motivo de obtener algún tipo de premio a cambio (Sánchez-Oliva et al., 2011) o por la penalización consecuente de no desarrollarlo (Deci y Ryan, 2000). 
Por último, dentro del continuo motivacional, se encuentra la desmotivación, que constituye un estado psicológico en que las personas no tienen ni un sentido de eficacia o de control en lo que respecta a la consecución de un resultado deseado.

Asimismo, para que exista algún tipo de motivación, una persona debe tener cubiertas tres necesidades psicológicas consideradas como básicas (Reinboth y Duda, 2006), o por el contrario, no tener frustradas estas tres necesidades fundamentales (Bartholomew, Ntoumanis, Ryan, y ThøgersenNtoumani, 2011). Estos tres mediadores esenciales se denominan: autonomía, competencia y afiliación. Concretamente, la frustración de autonomía es el sentimiento que tiene un árbitro para llevar a cabo su labor, no sintiéndose propietario de sus actos y de no guiar de forma deliberada su comportamiento. La frustración de competencia se define como la percepción que tiene el árbitro sobre su nivel de capacidad a la hora de desarrollar su función. No sintiéndose eficaz en la función que desempeña. Mientras que la afiliación, es la percepción de frustración del árbitro de no sentirse relacionado con otras personas durante la actividad desarrollada.

Asimismo, los árbitros deportivos han sido reportados a experimentar niveles relativamente altos de estrés asociados a sus labores. No obstante, pocos estudios han examinado el desarrollo del burnout, entendido éste como como una sensación de agotamiento emocional en el desarrollo de su trabajo.

De esta manera, el principal objetivo de esta investigación se centra en analizar los antecedentes motivacionales que encuentra un árbitro de baloncesto y su relación con el agotamiento emocional. Por tanto, acorde con el objetivo principal del estudio, las hipótesis que se plantean es que un árbitro con mayores niveles de autodeterminación y bajos niveles de frustración de las necesidades psicológicas básicas se relacionaría con altos niveles de eficacia profesional y bajo agotamiento emocional. Del mismo modo, la desmotivación sería el mayor predictor del agotamiento emocional.

\section{Método}

\section{Participantes}

La muestra de la investigación estuvo compuesta por 30 árbitros con edades comprendidas entre los 17 y los 41 años $(M$ = 24.8; DT = 5.74). Todos ellos arbitran en la comunidad de Extremadura (España)

\section{Instrumentos}

Frustración de las Necesidades Psicológicas Básicas: para la medición de la frustración que percibe un árbitro de sus necesidades psicológicas básicas se ha realizado una adaptación al castellano del cuestionario Psychological Need Thwarting
(PNTS: Bartholomew et al. 2011), utilizado y desarrollado en el ámbito educativo. Este instrumento está formado por 12 ítems y consta de tres factores como son percepción de frustración autonomía ( 4 ítems, ej.: Me siento presionado a comportarme de determinada manera), la percepción de frustración de competencia ( 4 ítems, ej.: Hay situaciones que me hacen sentir incompetente) y la percepción de frustración de relaciones sociales ( 4 ítems, ej.: Siento que los demás pueden ser indiferentes conmigo).

Regulación motivacional para arbitrar: para valorar los motivos que impulsan a los árbitros para ejercer la función de arbitrar, se ha utilizado la adaptación del cuestionario que valora la motivación autodeterminada en el contexto educativo, Autonomous Motivation for teaching (WMI: Work Motivation Inventory) (Blais, Lachance, Vallerand, Briere y Riddle, 1993), formado por 24 ítems divididos en 6 factores que son la regulación intrínseca ( 4 ítems, ej.: Porque obtengo mucho placer al aprender nuevas cosas), la regulación integrada (4 ítems, ej.: Porque es parte de la forma que he elegido para vivir mi vida), la regulación identificada 4 ítems ( 4 ítems, ej.: Porque quiero dedicar mi carrera profesional a esto), motivación introyectada (4 ítems, ej.: Porque quiero ser muy bueno, de lo contrario estaría muy decepcionado), la regulación externa (4 ítems, ej.: Porque me permite ganar dinero) y la desmotivación( 4 ítems, ej.: Me pregunto esta cuestión, no parezco capaz de manejar las decisiones importantes relacionadas con el arbitraje).

Burnout: para valorar el burnout en árbitros se ha utilizado una adaptación del cuestionario Maslach Burnout Inventory (MBI: Maslach y Jackson, 1986) de la versión traducida al castellano por Gil Monte, (2002), utilizado en el ámbito educativo. El cuestionario original está formado por tres factores: agotamiento emocional, eficacia profesional y cinismo, pero para el desarrollo de este trabajo se ha utilizado únicamente el factor del agotamiento emocional (3 ítems, ej.: Me encuentro agotado cuando me levanto y tengo que enfrentarme a un nuevo partido).

El formato de respuesta es una escala tipoLikert de 1 a 5 , donde 1 corresponde a totalmente en desacuerdo y el 5 a totalmente de acuerdo. Además, todos los factores han obtenido una consistencia interna recomendada

\section{Procedimiento}

En primer lugar, se contactó con la federación extremeña de baloncesto informándoles del objetivo del estudio. Tras su consentimiento, se les mandó un enlace para que lo reenviara a todos los árbitros de la federación. Por último, voluntariamente los árbitros accedían a la plataforma "Google Docs" para rellenar dicho cuestionario.

\section{Resultados}


Para el tratamiento de los datos se ha utilizado el software estadístico SPSS 19.0. En la tabla 1 aparecen los valores descriptivos y las correlaciones de las variables del estudio. En cuanto al análisis descriptivo, se observan medias elevadas en la motivación intrínseca y en la eficacia profesional. Respecto al análisis correlacional, se aprecian relaciones positivas y significativas entre la desmotivación y los tres factores de la frustación de las necesidades psicológicas básicas (autonomía, competencia y relaciones sociales), lo mismo ocurre con el agotamiento emocional que se correlaciona de forma positiva con la frustación de autonomía, competencia y relaciones sociales. Además, la desmotivación se asocia de forma positiva con el agotamiento emocional.

Tabla 1. Estadísticos descriptivos y correlaciones entre los factores del estudio

\begin{tabular}{lccccccccccccc}
\hline & $\mathrm{M}$ & $\mathrm{DT}$ & 2 & 3 & 4 & 5 & 6 & 7 & 8 & 9 & 10 \\
\hline 1.AUT & 1.80 & .89 & $.51^{* *}$ & $.78^{* *}$ & -.17 & -.02 & -.23 & .21 & .10 & $.421^{*}$ & $.367^{*}$ \\
2.COM & 1.58 & .74 & - & $.52^{* *}$ & -.09 & .34 & -.22 & .02 & .06 & $.438^{*}$ & $.492^{* *}$ \\
3.REL & 1,78 & .89 & - & - & -.25 & .05 & -.13 & .16 & .07 & $.401^{*}$ & $.405^{*}$ \\
4.INTRI & 4.16 & .53 & - & - & - & .29 & .26 & .09 & .16 & -0.07 & 0.16 \\
5.INTE & 3.31 & .95 & - & - & - & - & $.73^{* *}$ & $.52^{* *}$ & .34 & 0.06 & 0.25 \\
6.IDE & 3.12 & .84 & - & - & - & - & - & $.363^{*}$ & .29 & 0.31 & $.367^{*}$ \\
7.INTRO & 2.05 & .86 & - & - & - & - & - & - & $.56^{* *}$ & $.439^{*}$ & $.485^{* *}$ \\
8.EXT & 2.95 & .88 & - & - & - & - & - & - & - & 0.23 & 0.26 \\
9.DES & 1.77 & .73 & - & - & - & - & - & - & - & - & $.615^{* *}$ \\
10.AGO & 1.90 & .86 & - & - & - & - & - & - & - & - & - \\
\hline
\end{tabular}

Nota: AUT (Autonomía), COM (Competencia), REL (Relaciones Sociales), INTRI (Intrínseca), INTE (Integrada), IDE (Identificada), INTRO (Introyectada), EXT (Externa), DES (Desmotivación) y AGO (Agotamiento Emocional)

Con la finalidad de comprobar cual era el mejor predictor del agotamiento emocional, se realizó un análisis de regresión lineal por pasos, donde las variables independientes eran los factores de frustración de las necesidades psicológicas básicas y los factores de la regulación motivacional. En la tabla 2, se observa como sólo la desmotivación se presentó como un predictor significativo del agotamiento emocional, siendo el porcentaje de varianza explicada del 38\%. En este caso, dicha variable lo predice de forma positiva, es decir, cuanto mayor sea el agotamiento emocional mayor será la desmotivación por seguir realizando su labor arbitral.

Tabla 2. Coeficientes del análisis de regresión por pasos considerando como variable dependiente el Agotamiento Emocional

\begin{tabular}{llccc}
\hline Variable & $\beta$ & $R^{2}$ & $t$ & $P$ \\
Paso 1 & & .38 & & \\
Desmotivación & .61 & & 4.12 & .00 \\
\hline
\end{tabular}

\section{Discusión}

El objetivo de esta investigación era examinar los antecedentes motivacionales que encuentra un árbitro de baloncesto para desempeñar su función y su relación con el agotamiento emocional.

La hipótesis planteaba que un árbitro con mayores niveles de autodeterminación y bajos niveles de frustración de las necesidades psicológicas básicas se relacionaría de forma positiva con bajos niveles de agotamiento emocional. Del mismo modo, la desmotivación sería el mayor predictor del agotamiento emocional. De acuerdo con los resultados encontra- dos se puede afirmar, que los árbitros con mayores niveles de autodeterminación no van a sentir la sensación de estar "quemado" por la función que desarrolla, sin embargo, cuando las regulaciones motivacionales fluctúan en la inexistencia de motivaciones intrínseca y/o extrínsecas como es el caso de la desmotivación, un árbitro no encontraría sentido a lo que hace $y$, por tanto, se relacionaría de forma positiva con la sensación de agotamiento emocional. Hasta ahora, se habían hallado conclusiones en otros significativos deportivos de agotamiento emocional a causas debidas al aumento en la toma de decisiones (Conton, 1995), a fluctuaciones en tipos de liderazgo (Lobban, Husted, y Farewell, 1988), a limitacio- 
nes situacionales (Jex, 1998) y al descenso en el compromiso organizacional (Kalliath, O’ Driscoll y Gillespie, 1998), pero no se habían encontrado relaciones significativas entre las percepciones motivacionales con la sensación de agotamiento emocional, ya que son escasas las investigaciones que han abordado las posibles relaciones de estas variables.

Por el contrario, como adelantaban en el ámbito educativo en un estudio desarrollado con profesores de Educación Física, (Bartholomew et al., 2011), una baja frustración de las necesidades psicológicas básicas se relacionan positivamente con niveles altos de autodeterminación. En este estudio, una frustración en la necesidad psicológica básica de autonomía, competencia y relaciones sociales muestra un sentimiento de no saber por qué seguir arbitrando en la modalidad deportiva de baloncesto.

Asimismo, la desmotivación se considera el mayor predictor del agotamiento emocional; por lo que un árbitro desmotivado en la labor que realiza tendría prevalencia a estar quemado y, como han encontrado diferentes estudios en otros ámbitos (Ortega y López, 2004), sería un riesgo latente a abandonar la función de arbitrar.

Por último, es necesario tener en cuenta los antecedentes motivacionales de los árbitros, no sólo de baloncesto, sino de todas las disciplinas deportivas, porque es un colectivo necesario y fundamental para que sea posible la práctica deportiva.

\section{Referencias}

Bartholomew, K. J., Ntoumanis, N., Ryan, R.M., y Thøgersen-Ntoumani, C. (2011). Psychological need thwarting in the sport context: development and initial validation of a psychometric scale. Journal of Sport \& Exercise Psychology, 33, 75-102.

Blais, R., Lachance, L., Vallerand, J., Briere, M., y Riddle, S. (1993). L'inventaire des motivacions au travail. Reveu quèbècoise de psychologic, 14(3), 21-23.

Bruner, J.S. (1988). Desarrollo cognitivo y educación. Madrid: Morata.

Cotton, J.L. (1995). Participation's effect on performance and satisfaction: A reconsideration of Wagner. Academy of Management Review, 20, 276278.

Deci, E.L., y Ryan, R.M. (1985). Intrinsic motivation and self-determination in human behavior. New York: Plenum Press.

Deci. E. L., y Ryan, R. M. (2000). The "what" and "why" of goal pursuits: Human needs and the self-determination of behaviour. Psychological Inquiry, 11, 227-268.

Feu, S., Ibáñez, S., Graça, A., y Sampaio, J. (2007). Evaluación psicométrica del cuestionario de orientación de los entrenadores en una muestra de entrenadores españoles de balonmano. Psycothema, 19(4), 699-705.

Gil Monte, P.R. (2002). Validez de la adaptación al español del Maslach Burnout Inventory-General Survey. Salud Publica Mexicana, 44, 33-40.

Jex, S.M. (1998). Stress and job performance: Theory, research and implications for managerial practice. Thousand Oaks, CA: Sage Publications.

Kalliath, T.J., O’ Driscoll, M.P., y Gillespie, D.F. (1998). The relationship between burnout and organizational commitment in two samples of health professionals. Work \& Stress, 12, 179-185.
Lobban, R.K., Husted, J., y Farewell, V.T. (1998). A comparison of the effect of job demand, decision latitude, and supervisory style on selfreported job satisfaction. Work \& Stress, 12, 337-350.

Maslach, C., y Jackson, S.E. (1986). Maslach Burnout Inventory. Palo Alto, CA: Consulting Psychlogists Press.

Montero, A., Ezquerro, M., y Buceta, J.M. (2005). Variaciones de las conductas de los entrenadores infantiles a lo largo de la competición. Kronos, Rendimiento en el deporte, 4, 52-56.

Ortega, C., y López, F. (2004). El burnout o síndrome de "estar quemado" en los profesionales sanitarios: revisión y perspectivas. Revista Internacional de Psicología Clinica y de la Salud/ International Journal of Clinical and Health Psychology, 4, 137-160.

Reinboth, M., y Duda, J.L. (2006). Perceived motivational climate, need satisfaction and indices of well-being in team sports: a longitudinal perspective. Psychology of Sport and Exercise, 7, 269-286.

Sánchez-Oliva, D., Leo, F. M., Sánchez-Miguel, P. A., Gómez, F.R., y García-Calvo, T. (2011). Teoría de autodeterminación y comportamientos prosociales en jóvenes jugadores de fútbol. Apunts: Educación física y deportes, 103, 32-38.

Torregrosa, M., Cruz, J., Sousa, C., Viladrich, C., Villamarín, F., García Mas, A., y Palou, P. (2007).La influencia de padres y madres en el compromiso deportivo de futbolistas jóvenes. Revista Latinoamericana de Psicología, 39, 227-237. 